\title{
Cell dynamics with a novel phase referenced low-coherence interferometer with sub-wavelength and sub-hertz sensitivity
}

Changhuei Yang, Adam Wax, Kamran Badizadegan, Ramachandra R. Dasari, Michael S. Feld

Changhuei Yang, Adam Wax, Kamran Badizadegan, Ramachandra R. Dasari, Michael S. Feld, "Cell dynamics with a novel phase referenced lowcoherence interferometer with sub-wavelength and sub-hertz sensitivity," Proc. SPIE 4619, Coherence Domain Optical Methods in Biomedical Science and Clinical Applications VI, (14 June 2002); doi: 10.1117/12.470484

Event: International Symposium on Biomedical Optics, 2002, San Jose, CA, United States 


\title{
The Study of Cell Dynamics with a Novel Phase Referenced Low Coherence Interferometer with sub-wavelength and sub-hertz Sensitivity. Changhuei Yang, Adam Wax, Kamran Badizadegan, Ramachandra R. Dasari, Michael S. Feld Massachusetts Institute of Technology, Cambridge, MA 02139
}

\begin{abstract}
We report the use of a highly sensitive phase based motion measurement technique to study the correlation of cellular metabolic rate with cellular motions. The technique is based on a modified Michelson interferometer with a composite laser beam of $1550 \mathrm{~nm}$ low coherence light and $775 \mathrm{~nm}$ CW light. In this system, motional artifacts from vibrations in the interferometer are completely eliminated. We demonstrate that the system is sensitive to motions as small as $3.6 \mathrm{~nm}$ and velocities as small as $1 \mathrm{~nm} / \mathrm{s}$. Using the system, we show that the cellular motions are strongly dependent on the ambient temperature. We observe that the dependency does not conform to Brownian motion predictions but instead appears to correlate with the optical ambient temperature that the cells have evolved to operate in.
\end{abstract}

Keywords: coherence, interferometry, cell, dynamics, metabolic, phase, harmonic

\section{INTRODUCTION}

Detection of motions with amplitude less than an optical wavelength and/or velocity less than $1 \mu \mathrm{m} / \mathrm{s}$ is important for the study of subcellular dynamics in living biological tissues. Motions of such magnitude include the actin-based transport of organelles within the cells [1], the ruffling of cell membranes [2] and the reaction of cell volumes to hyper/hypotonicity [3]. These motions are of significant biomedical interest and their study can potentially lead to new diagnostic modalities. For example, it has been established that cells with high metastatic potential are significantly more motile than normal cells [4-7]. Cell dynamics is also of particular interest in the study of cell volume regulation mechanisms which are identified by measurement of cell volume changes [3, 8, 9].

At present, cellular motions are generally studied via sequential recordings of high power microscope images. Such studies have two major limitations. First, the ultimate sensitivity to length changes of such an imaging method is limited by the resolving power of the microscope. It is generally accepted that a microscope cannot resolve at a scale finer than a tenth of a micron or a tenth of an optical wavelength. While such sensitivity is sufficient for the study of pseudopodal motions [10, 11], it is inadequate for the study of cellular motions on finer scales.

Second, microscopy techniques are unsuited for detecting changes in the cellular thickness. Aside from single cellular organisms, macrophages, red blood cells and a few other cell types, cells in their natural states are typically attached to their neighboring cells by binding proteins such as those of the cadherin and adherin families [12]. Such bindings effectively immobilize the cells and prevent them from moving extensively in the image plane in the case of a cell culture. The only spatial degree of motional freedom for these cells is in the vertical plane. In fact, cells have evolved a whole range of functions that involves motions in the vertical plane. An example can be found in the case of secretary glands, where continuous exocytosis of hormone molecules via cell membrane budding processes occur on the outward facing cell membranes [13]. Such motions, which cause the upper cell membranes to fluctuate vertically, cannot be studied with conventional microscopy techniques.

One of the more extensively studied aspects of cellular motions is the field of cell volume regulation. Cells have evolved a whole range of volume regulation mechanisms, by which they maintain their cell volumes in changing external environments [3]. For example, a change in the osmolality of the external environment will cause an osmotic outflow of water from a cell. The cell compensates by opening appropriate ion channels within its cell membrane. The ensuing outflow of cytoplasmic salt ions 
equilibrates the osmotic pressure and allows the cell to recover its original cell volume in time. As we shall later see, this action and reaction translate to very slow and small changes in the cell height. The field of cell volume research has significant applications in the understanding and treatment of certain diseases. For example, the toxicity of cholera toxin arises from its ability to disrupt cell volume regulation mechanisms, thereby, causing a massive loss of fluids from a cell [13-16].

\section{EXPERIMENTAL SETUP}

In this section, we describe a novel phase-referenced low coherence interferometry (PRI) method, that is capable of measuring slow $(\sim 1-1000 \mathrm{~nm} / \mathrm{s})$ and small $(4 \mathrm{~nm}$ or more) motions. In addition, it provides depth resolution and can be used for tomographic imaging. This method is particularly suited for the study of cell membrane dynamics. We demonstrate that, with PRI, it is possible to track the cell membrane motions of a single or a few cells. Further, PRI can measure very small motions very accurately. Finally, PRI has the advantage that it is completely free of interferometer vibrations and drifts. This stability makes it possible to track the cell membrane dynamics over very long time scales. The experiment requires the sample to include a strong reflecting interface for the $\mathrm{CW}$ wavelength.

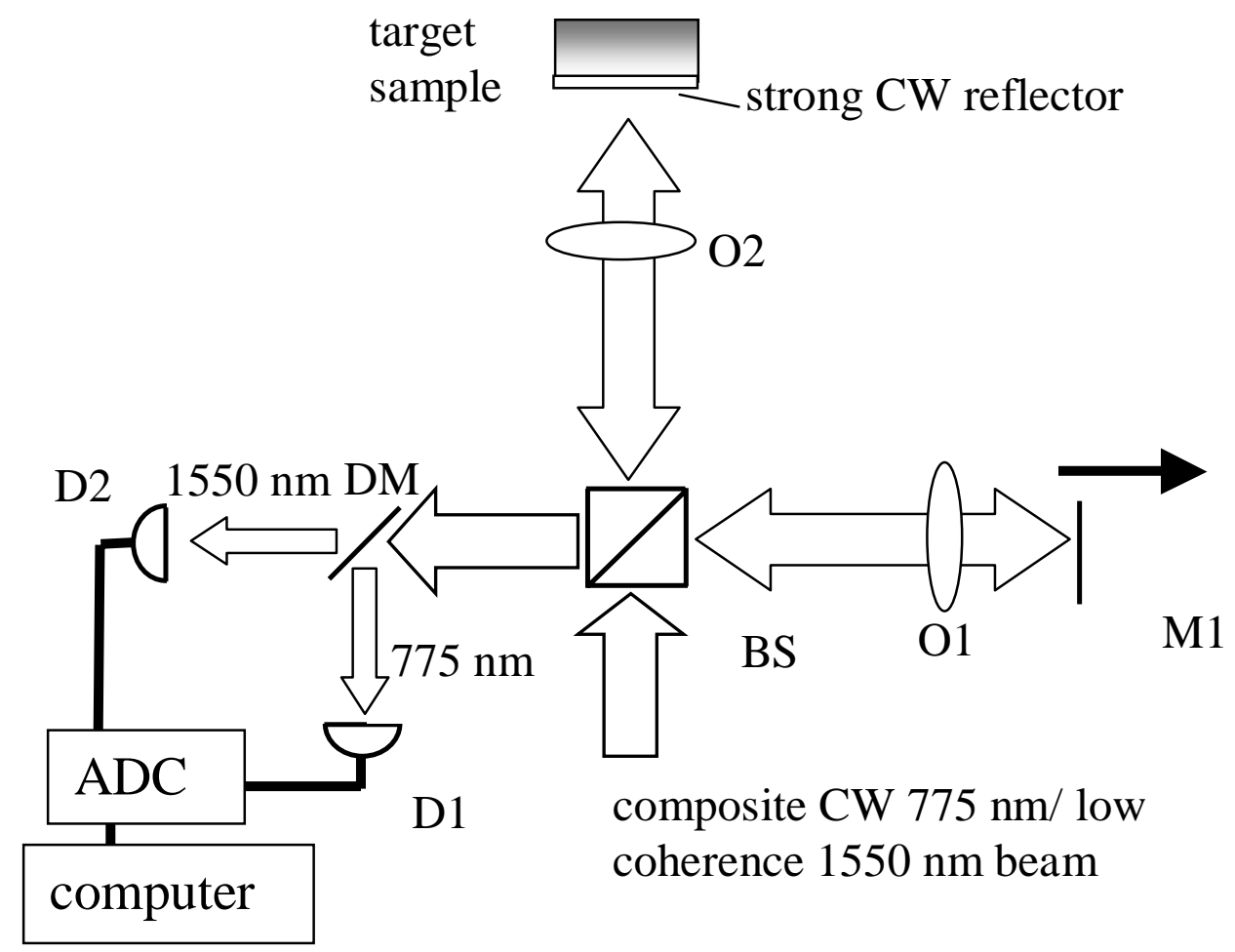

Figure 1. Experimental Setup. M1 is a reference mirror; BS is a beamsplitter; $\mathrm{D} 1,2$ are photodetectors; DM is a $775 \mathrm{~nm} / 1550 \mathrm{~nm}$ dichroic mirror, 01,2 are microscope objectives.

The input light to the system is a two-color composite beam composed of CW light from a Ti:sapphire laser at $775 \mathrm{~nm}$ and low coherence light (bandwidth $=40 \mathrm{~nm}$ ) from a $1550 \mathrm{~nm}$ Optospeed superluminescent diode. The composite beam is divided into two at the beamsplitter. One part (signal) is brought to a focus at the target sample, while the other is incident on a reference mirror moving at $0.5 \mathrm{~mm} / \mathrm{s}$, which induces a Doppler shift on the reference beam. The technique requires a strong reflection at the $\mathrm{CW}$ wavelength from a control interface at the sample. We fulfill this requirement by placing the sample on a glass cover slip; the index mismatch between the first glass interface and air is sufficient to provide the strong reflection. The powers of the $775 \mathrm{~nm}$ and $1550 \mathrm{~nm}$ beam components at the sample are $0.9 \mu \mathrm{W}$ and $1.2 \mu \mathrm{W}$, respectively. An IR objective lens (12 mm focal length) focuses the composite beam onto the sample with a FHWM of 
about $17 \mu \mathrm{m}$ at the $1550 \mathrm{~nm}$. The focusing effect of the lens on the CW beam is not important, as we do not rely on this wavelength for imaging.

The back-reflected beams are recombined at the beamsplitter, separated by their wavelength components using a dichroic mirror, and measured separately by photodetectors. The resulting heterodyne signals at their respective Doppler-shifted frequencies are measured and digitized by means of a 16-bit $100 \mathrm{kHz}$ A/D converter. The signals are bandpassed around their respective center heterodyne frequencies and Hilbert transformed, to extract their corresponding phases of the heterodyne signals, $\Psi_{C W}$ and $\Psi_{L C}$. (The subscripts $\mathrm{CW}$ and LC will be used throughout to denote the $775 \mathrm{~nm} \mathrm{CW}$ and $1550 \mathrm{~nm}$ low coherence wavelength components, respectively.)

\section{THE PRINCIPLE OF PRI}

We denote the length of the reference and signal interferometer arms as $x$ and $x_{I}$ respectively. The principle of PRI can be illustrated through the simple case where the sample consists of two reflecting interfaces, 1 and 2. The separation between them is $L$, and the refractive index of the material between them is $n$. We assume that the first interface is at the end of the signal interferometer arm. We further assume that the first interface is a strong reflector at the $\mathrm{CW}$ wavelength.

As we scan the reference mirror and change $x$, we will see a continuous heterodyne signal associated with the first interface that exists across the entire scan due to the $\mathrm{CW}$ light. As the first interface is assumed to be a strong reflector, the corresponding $\mathrm{CW}$ heterodyne signal associated with the second interface will be negligible. The continuous heterodyne signal will be anchored with respect to the position of the first interface. In other words, every scan of $x$ will have the same heterodyne phase where $x=x_{1}$. This allows the $\mathrm{CW}$ heterodyne signal to serve as a convenient ruler which position is fixed with reference to the first interface.

In the case of the low coherence light, we would see two low coherence heterodyne signal envelopes associated with the two interfaces in each scan. In these cases, every scan of $\mathrm{x}$ will have the same low coherence heterodyne phases where $x=x_{1}$ and where $x=x_{1}+n L$. The associated heterodyne signals are anchored to the positions of the interfaces.

A straightforward way to determine if the second interface has moved with respect to the first interface would be by assessing if the centers of these heterodyne envelops have displaced with respect to the each other. However, this approach is limited by the accuracy to which the envelope centers can be determined. In a typical experiment, the accuracy is about $1 \mu \mathrm{m}$. This approach will not be sensitive enough to measure small length changes. Fortunately, the displacement of the second interface will not only shift the associated envelope but also move the heterodyne phase.

As such, by measuring the low coherence heterodyne with respect to the $\mathrm{CW}$ heterodyne phase, we can determine the displacement of the second interface with respect to the first interface. Since such length measurement can be made to a small fraction of a wavelength, the sensitivity of the technique is very high. The above explanation does not account for the fact that the heterodyne signals measurements are made in time. In an ideal world where the interferometer is vibration free and where the translation of the reference mirror occurs at a constant velocity $v$, the time traces of the heterodyne signals can be recasted as functions of the reference arm length, $x$. The function can be derived simply from the relation that $x=v t$. In reality, neither of those assumptions holds. Fortunately, the vibration correction mechanism of harmonic phase based interferometer allows us to eliminate these effects from the measurements. To understand the correction that occurs in the PRI system, we shall now explain the principle in time domain.

We denote the rate of change of path length difference between the reference and signal arms by $v$. This term includes both the translation velocity of the reference mirror and the jitter of the interferometer. The phase of the $\mathrm{CW}$ heterodyne signal is given by:

$$
\begin{gathered}
\psi_{C W}(t)=\bmod _{2 \pi}\left(\arg \left(R_{C W, 1} e^{i k_{C W} 2 \int_{O}^{t} v d t}+R_{C W, 2} e^{i k_{C W} 2\left(\int_{0}^{t} v d t-n_{C W} L\right)}\right)\right) \\
\cong \bmod _{2 \pi}\left(\arg \left(R_{C W, 1} e^{i k} C W 2 \int_{0}^{t} v d t\right)\right)=\bmod _{2 \pi}\left(k_{C W} 2 \int_{0}^{t} v d t\right),
\end{gathered}
$$


with $R_{C W, j}$ the reflectivity of the interface $j$ at the $\mathrm{CW}$ wavelength, and $k$ the optical wavenumber. The factors of 2 in the exponents are due to the effective doubling of optical paths in the back reflection geometry. The approximation is valid provided that the first interface is a strong reflector compared to the second interface $\left(R_{C W, 1} \gg R_{C W, 2}\right)$. The term $\int_{0}^{t} v d t$ is equal to $\left(x-x_{1}\right)$ from the previous explanation.

The situation is different for the low coherence light. The LC heterodyne phase is given by:

$$
\begin{aligned}
& \psi_{L C}(t) \\
& =\bmod _{2 \pi}\left(\arg \left(\begin{array}{l}
R_{L C, 1} e^{i k} L C 2\left(\int_{0}^{t} v d t\right) e^{-\left(\frac{2}{l_{c}}\left(\int_{0}^{t} v d t\right)\right)^{2}}+ \\
\left.R_{L C, 2} e^{i k_{L C} 2\left(\int_{0}^{t} v d t-n_{L C} L\right)} e^{-\left(\frac{2}{l_{c}}\left(\int_{0}^{t} v d t-n_{L C} L\right)\right)^{2}}\right)
\end{array}\right)\right)
\end{aligned}
$$

with $l_{c}$ the coherence length. When $L$ is larger than the coherence length, the LC heterodyne signal trace will consists of two low coherence signals. The heterodyne signal associated with the second interface occurs when $\int_{O}^{t} v d t \approx n_{L C} L$. In that vicinity, $\psi_{L C}(t)$, will be dominated by the contribution from the light reflected by the second interface, since the contribution from the light reflected by the first interface is suppressed by coherence gating. $\int_{O}^{t} v d t \approx n_{L C} L$. The resulting phase of the low coherence signal associated with the second interface can be expressed as:

$$
\begin{aligned}
& \psi_{L C}(t) \\
& \cong \bmod _{2 \pi}\left(\arg \left(R_{L C, 2} e^{i k_{L C} 2\left(\int_{0}^{t} v d t-n_{L C} L\right)} e^{-\left(\frac{2}{l_{C}}\left(\int_{0}^{t} v d t-n_{L C} L\right)\right)^{2}}\right)\right) \\
& =\bmod _{2 \pi}\left(k_{L C} 2\left(\int_{0}^{t} v d t-n_{L C} L\right)\right)
\end{aligned}
$$

Given that the center wavelength of the low coherence source is chosen to be twice that of the CW source ( $2 k_{L C}=k_{C W}$ ), we can obtain a difference phase, $\psi_{D}$, that is simply a function of $L$ by subtracting twice of Eq. (3) from Eq. (1):

$$
\psi_{D}=\psi_{C W}-2 \psi_{L C}=\bmod _{2 \pi}\left(4 k_{L C} n_{L C} L\right)
$$

This subtraction yields the phase acquired by the low coherence light wave as it traverses from interface 1 to interface 2 and back. The effect of interferometer jitter, which is manifested as variations in $v$, is completely eliminated. By periodically scanning the sample and measuring this phase difference, changes in $L$ can be determined accurately. As the uncertainty in phase is about $4.5^{\circ}$, we can measure time dependent changes in $L$ with an accuracy of about $3.6 \mathrm{~nm}$. 

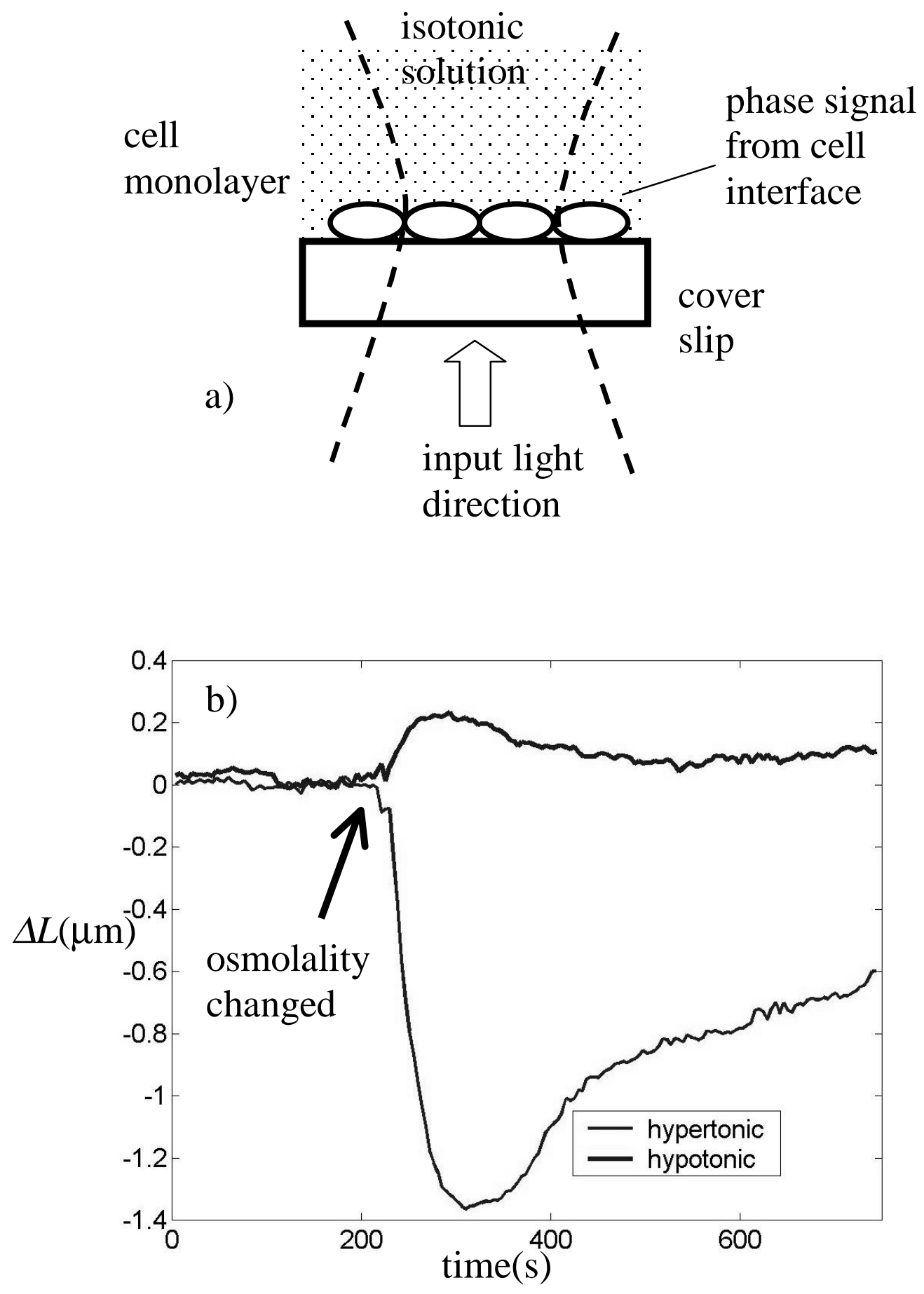

Figure 2. PRI analysis of a live HT29 cell monolayer grown on a cover slip. a) Experimental setup. The graph in b) shows changes in cell thickness when the osmolality is changed from its normal value to $85 \%$ and $115 \%$ at $t=230 \mathrm{~s}$. The maximum cell thickness changes are about $0.2 \mu \mathrm{m}$ and $-1.3 \mu \mathrm{m}$, respectively. The dashed lines indicate the beam profile. 
We apply the method to study the reaction of cell volume to hypo/hypertonic environment changes. It has been previously established using flow cytometry [8] that cells respond to hyper/hypotoncity with an initial shrinking/swelling followed by a compensatory, slower reversal of the initial responses.

We perform a similar experiment with HT29 intestinal epithelial cell monolayers grown to confluency on cover slips (Fig. 2a). The cell monolayers are immersed in isotonic buffer (Hank's balanced salt solution). We focus the low coherence beam directly on the cell culture. The index mismatch between the cells and the medium causes the top membrane surface to reflect light. The reflection from this interface cannot be resolved from the signal arising from the boundary between cover slip and cells as the optical path difference is smaller than the coherence length. This prevents extraction of amplitude-based information. However, phase-based information describing both of the two interfaces can still be extracted. The phase shift associated with either interface can be found by measuring at sufficient displacements from the peak interference signal. The focal spot is $17 \mu \mathrm{m}$ wide, and the average cell diameter is $10 \mu \mathrm{m}$, indicating that only a few cells are sampled during the experiment. A single cell sampling can be performed by choosing a tighter focal spot.

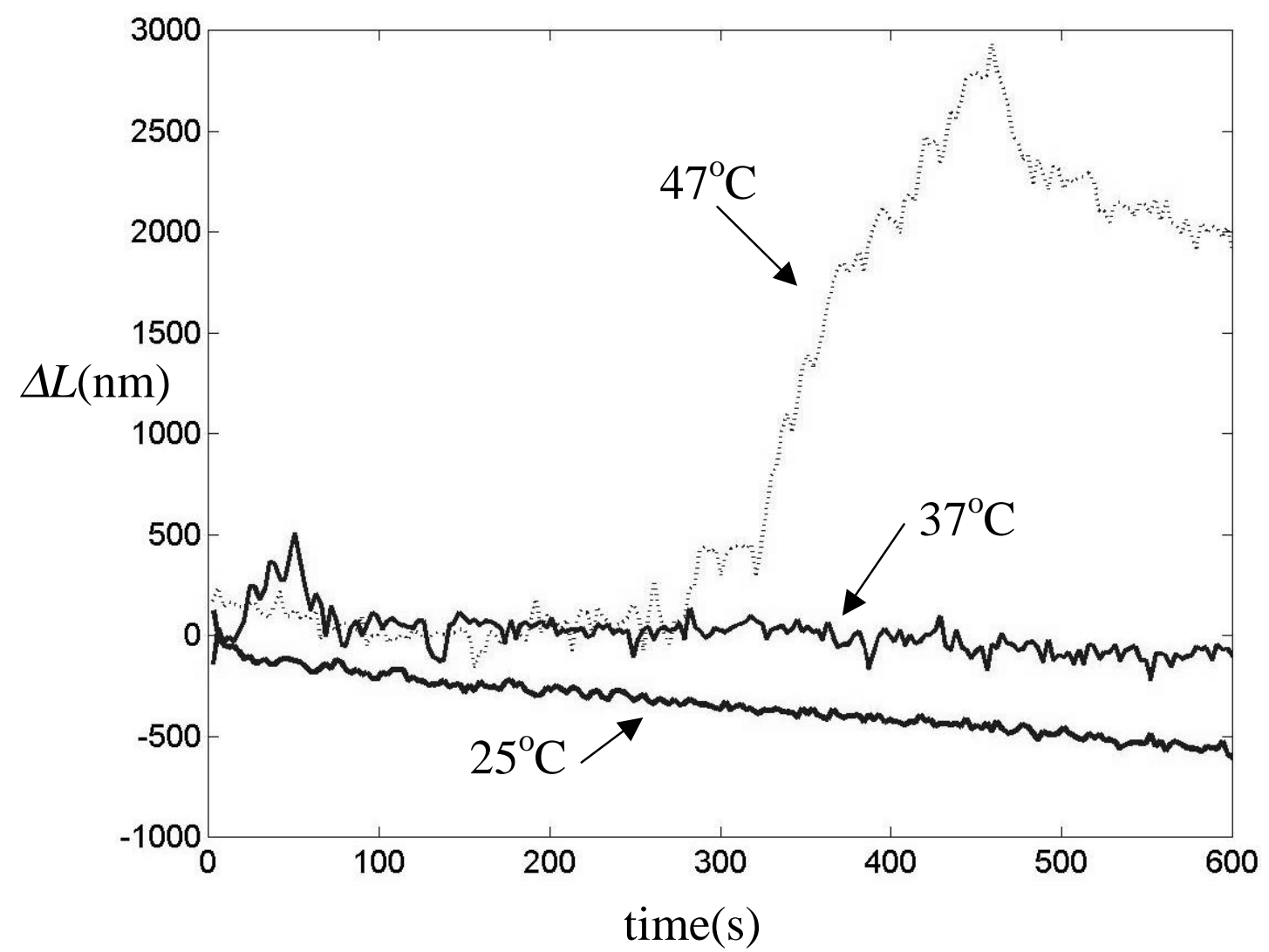

Figure 3. Plot of the experimental measurements of cell membranes fluctuations at different temperatures.

Upon abruptly changing the buffer's osmolality to $85 \%(115 \%)$ of its normal value, the phase shift $\psi_{D}$ associated with the cell membrane surface changes rapidly and then slowly recovers. In the hypotonic (hypertonic) case, the maximum change in cell thickness is about $0.2 \mu \mathrm{m}(-1.3 \mu \mathrm{m})$ (the refractive index of cells is assumed to be 1.36), and the duration of the entire process is several hundred seconds (Fig. 2b).

We note here that the compensatory reaction to the hypertonic change appears to be slower and weaker than the reaction to the hypotonic change. This is consistent with our predictions based on the complexity of the compensatory mechanisms. 
To our knowledge, this is the first time that cell volume changes of such small magnitudes have been observed optically and continuously traced in situ for a few cells. One of the consequences of this ability to observe the motions of a few cells is that we can begin to detect metabolically driven motions of cell membranes.

The cell membrane is held in place by a cytoskeleton scaffolding. This cytoskeleton framework is a highly dynamic structure. Its primary building blocks are actin filaments, which are stacks of actin monomers. These filaments are unstable structures and they maintain their length through a process known as treadmilling. By hydrolyzing ATP, actin monomers are added to one end of the filament to replace monomers that are constantly being lost at the other end. The energy dependency of the cytoskeleton suggests that the agitation level of the cell membrane will likely correlate to the ATP level and various chemical reaction rates in the cell.

We believe the relatively fast and small fluctuations of the trace in Fig. 2b, reflect the normal fluctuation of the cell membranes as the cytoskeleton undergoes metabolically driven changes. These observed fluctuations are about ten times above our noise limits.

\section{Correlating cell membrane fluctuations with cell metabolic level}

The establishment of a correlation between cell membrane fluctuations and cell metabolic level can lead to some important applications. At present, no means exists for determining the metabolic rates of cells on a single cell basis. To measure the metabolic rates of cells, a large number of cells is typically observed. In such a case, the oxygen intake and carbon dioxide output are sufficiently large for measurement purposes. From these measurements, the metabolic rates of the cells can be determined. As with all ensemble averaged based measurements, such a determination does not allow observation of variation on an individual cell level.

Since the PRI technique can be applied to observe a single cell, a correlation of membrane fluctuation to metabolic rate will permit a cell biologist to assess cell activity on a cell-by-cell basis.

Prompted by this motivation and the results in the above section, we performed several experiments to probe the correlation of cell membrane fluctuations with cell metabolic level.

In the first set of experiments, the ambient temperature of the cells' environment is changed. As with the previous experiment, we perform the experiments with HT29 intestinal epithelial cell monolayers grown to confluency on cover slips. As this cell line is derived from human tissue, we expect the cells to be optimized for operations at $37^{\circ} \mathrm{C}$ - the normal body temperature. We expect the metabolic rates to be lowered at other temperatures where proteins will not operate at their optimal rates.

The observed membrane fluctuations at $27^{\circ} \mathrm{C}, 37^{\circ} \mathrm{C}$ and $47^{\circ} \mathrm{C}$ are plotted in Fig. 3. The difference in mean fluctuation at $27{ }^{\circ} \mathrm{C}$ from those at the other temperatures is obvious. This correlation with temperature suggests that the fluctuation is indeed metabolically related. At present, more detailed experiments into this topic are being planned.

Another way by which we can alter the metabolic rates is by adding a toxin that disrupt the metabolic processes to the cells. The deprivation of ATP in the cell should cause the actin filaments to unravel. We should expect to see much less fluctuations. At the same time, we should expect to see a gradual fall in cell height. We can perform such an experiment by adding sodium azide - a potent poison, to a cell culture. We indeed observe a cessation of small fluctuation upon poisoning the cells. In addition, the membrane level appears to fall as predicted by our theory. Further experiments are plan ned along this line of investigation.

\section{Conclusions}

We have demonstrated that PRI is capable of measuring small and slow changes and that, in a biological context, it can be employed to study sub-cellular dynamics of a few cells or even parts of a single cell. The later can be achieved simply by employing a tighter focal spot through the use of higher power microscope objective. As with all phase-based techniques, PRI has to contend with the problem of phase wrapping. This phase ambiguity sets a limit on the fastest length change detectable. On the other hand, there is no theoretical lower limit to the dynamics which can be measured with this technique. This consideration makes PRI a good complement to Doppler OCT, in that together they can potentially measure motions over a wide range of magnitudes. 
The capability of PRI to study sub-cellular dynamics, in particular the fluctuations of cell membranes, can have important cell biology applications. For example, if the fluctuation rates are correlated with the metabolic rates of the cells, PRI can be applied as a means to measure the metabolic level of biological targets on a cell-by-cell basis. Given that cancerous cells have heightened metabolic rates, a system based PRI can potentially be used to assess to assess the progression of cancerous development on a single cell.

\section{References}

1. $\quad$ Rogers, S.L. and V.I. Gelfand, Membrane trafficking, organelle transport, and the cytoskeleton. Current Opinion in Cell Biology, 2000. 12(1): p. 57-62.

2. $\quad$ Coffey, D.S., Molecular and cellular biology of prostate cancer. 1991: Plenum Press.

3. Strange, K., Cellular and molecular physiology of cell volume regulation. 1993: CRC-Press.

4. Partin, A.W., J.S. Schoeniger, J.L. Mohler, and D.S. Coffey, Fourier-Analysis of Cell Motility Correlation of Motility with Metastatic Potential. Proceedings of the National Academy of Sciences of the United States of America, 1989. 86(4): p. 1254-1258.

5. Mohler, J.L., A.W. Partin, and D.S. Coffey, Prediction of Metastatic Potential by a New Grading System of Cell Motility - Validation in the Dunning-R-3327 Prostatic Adenocarcinoma Model. Journal of Urology, 1987. 138(1): p. 168-170.

6. Mohler, J.L., A.W. Partin, J.T. Isaacs, and D.S. Coffey, Metastatic Potential Prediction by a Visual Grading System of Cell Motility - Prospective Validation in the Dunning R-3327 Prostatic Adenocarcinoma Model. Cancer Research, 1988. 48(15): p. 4312-4317.

7. Partin, A.W., J.L. Mohler, and D.S. Coffey, Assessment of Metastatic Potential by Visual and Computerized Quantitative Grading Systems of Cancer Cell Motility. Cell Motility and the Cytoskeleton, 1988. 10(1-2): p. 345-345.

8. Hallows, K.R., C.H. Packman, and P.A. Knauf, Acute Cell-Volume Changes in Anisotonic Media Affect F-Actin Content of Hl-60 Cells. American Journal of Physiology, 1991. 261(6): p. C1154C1161.

9. Hallows, K.R., F.Y. Law, C.H. Packman, and P.A. Knauf, Changes in cytoskeletal actin content, $F$-actin distribution, and surface morphology during HL-60 cell volume regulation. Journal of Cellular Physiology, 1996. 167(1): p. 60-71.

10. Fukui, Y., T.Q.P. Uyeda, C. Kitayama, and S. Inoue, How well can an amoeba climb? Proceedings of the National Academy of Sciences of the United States of America, 2000. 97(18): p. 10020-10025.

11. Roberts, T.M., E.D. Salmon, and M. Stewart, Hydrostatic pressure shows that lamellipodial motility in Ascaris sperm requires membrane-associated major sperm protein filament nucleation and elongation. Journal of Cell Biology, 1998. 140(2): p. 367-375.

12. Lodish, H., Molecular Cell Biology. 4th ed. 1999, New York: W. H. Freeman and Co.

13. Dempsey, G.P., Bullivan.S, and W.B. Watkins, Ultrastructure of Rat Posterior Pituitary-Gland and Evidence of Hormone Release by Exocytosis as Revealed by Freeze-Fracturing. Zeitschrift Fur Zellforschung Und Mikroskopische Anatomie, 1973. 143(4): p. 465-484.

14. Low, S.Y., M.J. Rennie, and P.M. Taylor, Signaling elements involved in amino acid transport responses to altered muscle cell volume. Faseb Journal, 1997. 11(13): p. 1111-1117.

15. Orlov, S.N., J. Tremblay, and P. Hamet, cAMP signaling inhibits dihydropyridine-sensitive Ca2+ influx in vascular smooth muscle cells. Hypertension, 1996. 27(3): p. 774-780.

16. Rudolph, S.A., D.E. Schafer, and P. Greengard, Effects of Cholera Enterotoxin on Cation Fluxes, Cell-Volume, and Cyclic-Amp Levels in Turkey Erythrocyte. Biophysical Journal, 1976. 16(2): p. A171-A171. 